\title{
Presentations of Trivial Extensions of Finite Dimensional Algebras and a Theorem of Sheila Brenner
}

\author{
Elsa A. Fernández \\ Facultad de Ingeniería, Universidad Nacional de la Patagonia San Juan Bosco, \\ 9120 Puerto Madryn, Argentina \\ and \\ María Inés Platzeck ${ }^{1}$ \\ Departamento de Matemática, Universidad Nacional Del Sur, \\ 8000 Bahía Blanca, Argentina \\ Communicated by Kent R. Fuller \\ Received May 29, 2000 \\ DEDICATED TO IDUN REITEN ON HER 60TH BIRTHDAY \\ Let $\Lambda$ be a finite dimensional algebra over an algebraically closed field such that \\ any oriented cycle in the ordinary quiver of $\Lambda$ is zero in $\Lambda$. We describe the ordinary \\ quiver and relations for $T(\Lambda)=\Lambda \ltimes D(\Lambda)$, the trivial extension of $\Lambda$ by its minimal \\ injective cogenerator $D(\Lambda)$, and also for the repetitive algebra $\widehat{\Lambda}$ of $\Lambda$. Associated \\ with this description we give an application of a theorem of Sheila Brenner. $\odot 2002$ \\ Elsevier Science (USA)
}

\section{INTRODUCTION}

A finite dimensional $k$-algebra $\Lambda$ (associative, with identity) over an algebraically closed field $k$ is called self-injective if all projective $\Lambda$-modules are injective. An important class of self-injective algebras is formed by the

\footnotetext{
${ }^{1}$ The second author is a researcher from CONICET, Argentina.
} 
symmetric algebras. Recall that an algebra $\Lambda$ is said to be symmetric if $\Lambda \simeq D(\Lambda)$ as two sided $\Lambda$-modules.

Important and interesting examples of symmetric algebras are provided by group algebras of finite groups over fields and trivial extensions of finite dimensional algebras. Denote by $T(\Lambda)=\Lambda \ltimes D(\Lambda)$ the trivial extension of $\Lambda$ by its minimal injective cogenerator $D(\Lambda)$. We assume that $\Lambda$ is basic and indecomposable, so $\Lambda$ is given as the path algebra of a connected finite quiver $Q$ modulo an ideal of relations $I$.

In this work we will describe the ordinary quiver and relations for $T(\Lambda)$, under the assumption that any oriented cycle in the ordinary quiver of $\Lambda$ is zero in $\Lambda$. From this we deduce a description of the ordinary quiver and relations for $\widehat{\Lambda}$, the repetitive algebra of $\Lambda$.

The key point in the study of the ordinary quiver of $T(\Lambda)$ is showing that $Q_{T(\Lambda)}$ is obtained from $Q_{\Lambda}$ by adding $t$ arrows, where the number $t$ is equal to the dimension of $\operatorname{soc}(\Lambda)$, considered as module over the enveloping algebra $\Lambda^{e}$. We illustrate here the situation when $\Lambda$ is schurian. In this case we can choose a $k$-basis for the $\Lambda^{e}$-socle of $\Lambda$ consisting of maximal nonzero paths. For each of these we add an arrow to $Q_{\Lambda}$, and in the opposite direction. In this way, all arrows are in oriented cycles. The relations are particularly interesting in the case when the ordinary quiver of $\Lambda$ has no oriented cycles and parallel paths in $Q_{\Lambda}$ are equal in $\Lambda$, because they can be formulated directly in terms of the cycles in $k Q_{T(\Lambda)}$, independently of the relations for $\Lambda$. More precisely, the ideal $I_{T(\Lambda)}$ of relations for $T(\Lambda)$ is generated by

(i) the paths consisting of $n+1$ arrows in a cycle of length $n$,

(ii) the paths whose arrows do not belong to a single cycle, and

(iii) the difference $q-q^{\prime}$ of paths $q, q^{\prime}$ with the same origin and endpoint and having a common supplement in cycles of $Q_{T(\Lambda)}$. By a supplement of $q$ in the cycle $C$ we mean the path consisting of the remaining arrows of $C$.

This particular case has been crucial for the classification of all trivial extensions of finite representation type, and consequently for the study of iterated tilted algebras of Dynkin type, as done in the first author's doctoral dissertation $[\mathrm{F}]$, which will be published elsewhere.

The general case considered in this paper is stated in Theorem 3.9. Though technically more complicated, the essential ideas are contained in the above description.

Finally, we give an application of a theorem of Sheila Brenner. More precisely, Brenner shows in [B] how to determine the number of indecomposable direct summands of the middle term of an almost split sequence starting with a simple module. As a consequence of this result she obtains, for a self-injective artin algebra, the number of indecomposable direct 
summands of $\mathfrak{r} P / \operatorname{soc} P$, where $P$ is indecomposable projective. In general, it is not easy to compute these numbers for a given algebra. We give here a very simple interpretation of them in the particular case of the trivial extension $T(\Lambda)=\Lambda \ltimes D \Lambda$, where $\Lambda$ is an algebra such that any oriented cycle in $Q_{\Lambda}$ is zero in $\Lambda$. Our description is given in terms of oriented cycles in the quiver $k Q_{T(\Lambda)}$.

\section{PRELIMINARIES}

In this section we fix some notation and recall some relevant definitions and results which will be needed in the next sections. For a general reference in representation theory, we refer the reader to [ARS].

Throughout this paper $k$ will denote an algebraically closed field. By an algebra we mean a finite dimensional $k$-algebra which we will also assume to be basic and indecomposable. Thus $\Lambda \simeq k Q_{\Lambda} / I$, where $Q_{\Lambda}$ is a finite connected quiver and the ideal $I$ is admissible.

We denote by $\bmod \Lambda$ the category of finitely generated left $\Lambda$-modules, and by $D: \bmod \Lambda \rightarrow \bmod \Lambda^{o p}$ the standard duality $\operatorname{Hom}_{k}(\cdot, k)$. Also, we denote the Jacobson radical of $\Lambda$ by $\operatorname{rad} \Lambda$ or simply by $\mathfrak{r}$.

For a given quiver $Q$, we will denote by $Q_{0}$ the set of vertices, and by $Q_{1}$ the set of arrows between vertices. For each arrow $\alpha, s(\alpha)$ and $e(\alpha)$ will denote the start and end vertices of $\alpha$, respectively.

For each $i$ in $Q_{0}, S_{i}$ will be the simple $\Lambda$-module associated to $i$, and $P_{i}$ and $I_{i}$ will denote the projective cover and injective envelope of $S_{i}$, respectively. Thus, if $e_{i}$ is the idempotent element of $\Lambda$ corresponding to the vertex $i$ of $Q$, then $P_{i}=\Lambda e_{i}$.

Recall that the trivial extension $T(\Lambda)=\Lambda \ltimes D(\Lambda)$ of $\Lambda$ by $D(\Lambda)$ is the algebra with underlying vector space $\Lambda \oplus D(\Lambda)$, and the product is defined by $(\lambda, f)(\mu, g)=(\lambda \mu, \lambda g+f \mu)$ for any $\lambda, \mu \in \Lambda$.

We will need the following known facts, whose proof is straightforward.

Proposition 1.1. Let $\Lambda$ be an algebra. Then

(i) $\operatorname{rad} T(\Lambda)=(\mathfrak{r}, D(\Lambda))$, where $\mathfrak{r}$ denotes the radical of $\Lambda$.

(ii) $\operatorname{rad}^{2} T(\Lambda)=\left(\mathfrak{r}^{2}, \mathfrak{r} D(\Lambda)+D(\Lambda) \mathfrak{r}\right)$.

(iii) $\operatorname{rad} T(\Lambda) / \operatorname{rad}^{2} T(\Lambda)$ and $\left(\mathfrak{r} / \mathfrak{r}^{2}, D(\Lambda) /(\mathfrak{r} D(\Lambda)+D(\Lambda) \mathfrak{r})\right)$ are isomorphic vector spaces.

\section{THE ORDINARY QUIVER OF $T(\Lambda)$}

In this section we describe the ordinary quiver of $T(\Lambda)$ for any finite dimensional $k$-algebra $\Lambda$. 
Let $\Lambda=k Q_{\Lambda} / I_{\Lambda}$ be a fixed presentation for $\Lambda$. Given an element $x$ in $k Q_{\Lambda}$, we will denote by $\bar{x}$ the corresponding element in $k Q_{\Lambda} / I_{\Lambda}$.

For our purpose the following preliminary result will be useful.

LEMma 2.1. There is a short exact sequence of $\Lambda$-bimodules

$$
0 \rightarrow D(\Lambda) \mathfrak{r}+\mathfrak{r} D(\Lambda) \rightarrow D(\Lambda) \rightarrow D\left(\operatorname{soc}_{\Lambda^{e}} \Lambda\right) \rightarrow 0,
$$

where $\Lambda^{e}$ is the enveloping algebra of $\Lambda$.

Proof. Since $D\left(\operatorname{soc}_{\Lambda^{e}} \Lambda\right)=\operatorname{top}_{\Lambda^{e}}(D(\Lambda))$, there is a short exact sequence

$$
0 \rightarrow \operatorname{rad}_{\Lambda^{e}} D(\Lambda) \rightarrow D(\Lambda) \rightarrow D\left(\operatorname{soc}_{\Lambda^{e}} \Lambda\right) \rightarrow 0 .
$$

We only have to describe $\operatorname{rad}_{\Lambda^{e}} D(\Lambda)$. Since

$$
\operatorname{rad}\left(\Lambda^{e}\right)=\mathfrak{r} \otimes \Lambda^{o p}+\Lambda \otimes \mathfrak{r}^{o p},
$$

we have $\operatorname{rad}_{\Lambda^{e}} D(\Lambda) \simeq \operatorname{rad} \Lambda^{e} \cdot D(\Lambda) \simeq \mathfrak{r} D(\Lambda)+D(\Lambda) \mathfrak{r}$.

Proposition 2.2. If $\Lambda$ is an algebra with ordinary quiver $Q_{\Lambda}$, then the ordinary quiver of $T(\Lambda)$ is given by

(i) $\left(Q_{T(\Lambda)}\right)_{0}=\left(Q_{\Lambda}\right)_{0}$,

(ii) $\left(Q_{T(\Lambda)}\right)_{1}=\left(Q_{\Lambda}\right)_{1} \cup\left\{\beta_{p_{1}}, \ldots, \beta_{p_{t}}\right\}$, where $\left\{\bar{p}_{1}, \ldots, \bar{p}_{t}\right\}$ is a $k$-basis for $\operatorname{soc}_{\Lambda^{e}} \Lambda$, and for each $i, \beta_{p_{i}}$ is an arrow from $e\left(p_{i}\right)$ to $s\left(p_{i}\right)$.

Proof. (i) Let $Q_{0}=\{1,2, \ldots, n\}$ be the set of vertices of $Q_{\Lambda}$ and let $\left\{e_{1}, \ldots, e_{n}\right\}$ be the set of trivial paths in $k Q_{\Lambda}$. It is easy to verify that $\left\{\left(\overline{e_{1}}, 0\right), \ldots,\left(\overline{e_{n}}, 0\right)\right\}$ is a complete set of primitive orthogonal idempotents in $T(\Lambda)$. So, $Q_{T(\Lambda)}$ has $n$ vertices in one-to-one correspondence with $\left(\overline{e_{1}}, 0\right), \ldots,\left(\overline{e_{n}}, 0\right)$.

(ii) For each pair of integers $i, j$, with $1 \leq i, j \leq n$, the number of arrows from $i$ to $j$ is equal to $\operatorname{dim}_{k}\left(\left(\overline{e_{j}}, 0\right) \operatorname{rad} T(\Lambda) / \operatorname{rad}^{2} T(\Lambda)\left(\overline{e_{i}}, 0\right)\right)$. By Proposition 1.1, we have

$$
\begin{aligned}
& \operatorname{dim}_{k}\left(\left(\overline{e_{j}}, 0\right) \operatorname{rad} T(\Lambda) / \operatorname{rad}^{2} T(\Lambda)\left(\overline{e_{i}}, 0\right)\right) \\
& \quad=\operatorname{dim}_{k}\left(\overline{e_{j}} \mathfrak{r} / \mathfrak{r}^{2} \overline{e_{i}}\right)+\operatorname{dim}_{k}\left(\overline{e_{j}}(D(\Lambda) /(\mathfrak{r} D(\Lambda)+D(\Lambda) \mathfrak{r})) \overline{e_{i}}\right) .
\end{aligned}
$$

The first summand is equal to the number of arrows from $i$ to $j$ in $Q_{\Lambda}$. According to Lemma 2.1, the second summand is equal to the dimension of the subspace of $\operatorname{soc}_{\Lambda^{e}} \Lambda$ generated by all elements $\bar{p}_{k}$, where $p_{k}$ is a $k$-linear combination of paths starting at $j$ and ending at $i$.

Remark 2.3. We say that a path $q$ in $Q_{\Lambda}$ is maximal if $\bar{q} \neq 0$ and $\overline{\alpha q}=$ $0=\overline{q \alpha}$, for any arrow $\alpha$ in $\left(Q_{\Lambda}\right)_{1}$. Note that if $q$ is a maximal path, then $\bar{q} \in \operatorname{soc}_{\Lambda^{e}} \Lambda$. 
When $\Lambda$ is a schurian algebra, which means $\operatorname{dim}_{k} \operatorname{Hom}_{\Lambda}\left(P, P^{\prime}\right) \leq 1$ for every pair of indecomposable projective $\Lambda$-modules $P, P^{\prime}$, we can also give another description of $D(\Lambda) /(\mathfrak{r} D(\Lambda)+D(\Lambda) \mathfrak{r}) \simeq D\left(\operatorname{soc}_{\Lambda^{e}} \Lambda\right)$. In this case we have the following result:

Lemma 2.4. Let $\Lambda$ be a schurian algebra. Then $\operatorname{soc}_{\Lambda^{e}} \Lambda$ is the subspace of $\Lambda$ generated by all $\bar{q} \in \Lambda$, with q a maximal path in $Q_{\Lambda}$.

We now give some examples to illustrate the construction of $Q_{T(\Lambda)}$.

EXAMPLE 2.5. Let $\Lambda$ be given by the quiver

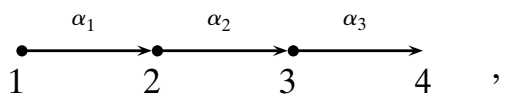

with the relation $\alpha_{3} \alpha_{2} \alpha_{1}=0$. Then $\left\{\bar{p}_{1}=\overline{\alpha_{2} \alpha_{1}}, \bar{p}_{2}=\overline{\alpha_{3} \alpha_{2}}\right\}$ is a $k$-basis for $\operatorname{soc} \Lambda$. So, according to Proposition 2.2, $Q_{T(\Lambda)}$ is the quiver

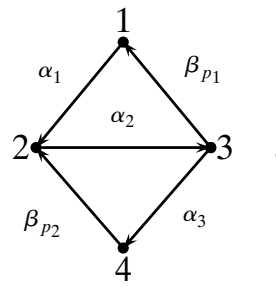

EXAMPLE 2.6. Let $\Lambda$ be given by the quiver

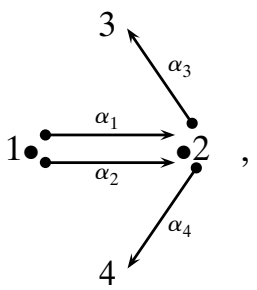

with relations $\alpha_{3} \alpha_{1}=\alpha_{3} \alpha_{2}, \alpha_{4} \alpha_{1}=\alpha_{4} \alpha_{2}$. 
In this case, $\left\{\bar{p}_{1}=\overline{\alpha_{1}-\alpha_{2}}, \bar{p}_{2}=\overline{\alpha_{3} \alpha_{1}}, \bar{p}_{3}=\overline{\alpha_{4} \alpha_{1}}\right\}$ is a $k$-basis for $\operatorname{soc} \Lambda$. Then $Q_{T(\Lambda)}$ is the following quiver:

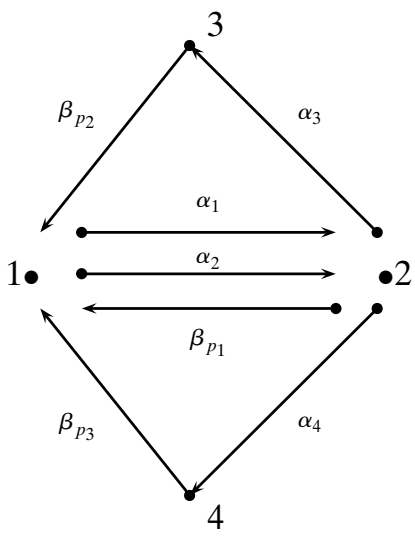

\section{THE RELATIONS FOR $T(\Lambda)$}

From now on we will assume that any oriented cycle in $Q_{\Lambda}$ is zero in $\Lambda$. In particular, the class of schurian algebras has this property.

Notation. In all that follows, we fix a set $\mathbb{M}=\left\{p_{1}, \ldots, p_{t}\right\}$ of elements in $k Q_{\Lambda}$ such that $\left\{\bar{p}_{1}, \ldots, \bar{p}_{t}\right\}$ is a basis for $\operatorname{soc}_{\Lambda^{e}} \Lambda$. Moreover, let $\left\{\bar{p}_{1}, \ldots, \bar{p}_{t}, \ldots, \bar{p}_{d}\right\}$ be a basis of $\Lambda$. We will denote by $\left\{\bar{p}_{1}{ }^{*}, \ldots, \bar{p}_{d}{ }^{*}\right\}$ the dual basis in $D(\Lambda)$.

Our next goal is to describe the ideal $I_{T(\Lambda)}$ of relations for $T(\Lambda)$. This will require some preliminary definitions and remarks.

Definition 3.1. Let $\mathbb{C}$ be an oriented cycle in $Q_{T(\Lambda)}$. We say that $\mathbb{C}$ is elementary if $\mathbb{C}=\alpha_{j} \cdots \alpha_{1} \beta_{p} \alpha_{n} \cdots \alpha_{j+1}$, with $\alpha_{1}, \ldots, \alpha_{n} \in\left(Q_{\Lambda}\right)_{1}$ and $p \in \mathbb{M}$ and $\bar{p}^{*}\left(\overline{\alpha_{n} \cdots \alpha_{1}}\right) \neq 0$. In this case, the weight of $\mathbb{C}$ is $w(\mathbb{C})=$ $\bar{p}^{*}\left(\overline{\alpha_{n} \cdots \alpha_{1}}\right) \in k^{*}$.

EXAMPLE 3.2. Let $\Lambda$ be the algebra given in Example 2.6 and consider the following $k$-basis for $\Lambda$ :

$$
\left\{\overline{p_{1}}=\overline{\alpha_{1}-\alpha_{2}}, \overline{p_{2}}=\overline{\alpha_{3} \alpha_{1}}, \overline{p_{3}}=\overline{\alpha_{4} \alpha_{1}}, \overline{\alpha_{1}}, \overline{\alpha_{3}}, \overline{\alpha_{4}}, \overline{e_{1}}, \overline{e_{2}}, \overline{e_{3}}, \overline{e_{4}}\right\} .
$$

Since ${\overline{p_{1}}}^{*}\left(\overline{\alpha_{1}}\right)=\overline{\alpha_{1}-\alpha_{2}} *\left(\overline{\alpha_{1}}\right)=0$, the oriented cycle $\beta_{p_{1}} \alpha_{1}$ is not an elementary cycle. Now, it is not difficult to check that the oriented cycles

$$
\beta_{p_{2}} \alpha_{3} \alpha_{1}, \beta_{p_{3}} \alpha_{4} \alpha_{1}, \beta_{p_{2}} \alpha_{3} \alpha_{2}, \beta_{p_{3}} \alpha_{4} \alpha_{2} \text {, and } \beta_{p_{1}} \alpha_{2}
$$

are all the elementary cycles in $Q_{T(\Lambda)}$, up to cyclic permutations. 
In all that follows, when we say that a path $q$ is contained in the path $q^{\prime}$, it will be understood that $q^{\prime}=\gamma_{2} q \gamma_{1}$, where $\gamma_{1}, \gamma_{2}$ are paths with $e\left(\gamma_{1}\right)=s(q)$ and $s\left(\gamma_{2}\right)=e(q)$.

Remark 3.3. If $0 \neq \bar{v} \in \Lambda$, then there are paths $\delta_{1}, \delta_{2}$ in $k Q_{\Lambda}$ and $p_{j} \in \mathbb{M}$ such that $\bar{p}_{j}^{*}\left(\overline{\delta_{1} v \delta_{2}}\right) \neq 0$, and in particular, any nonzero path in $\Lambda$ is contained in an elementary cycle.

In fact, let $\delta_{1}, \delta_{2}$ be paths in $k Q_{\Lambda}$ of maximal length such that $\bar{z}=$ $\overline{\delta_{1} v \delta_{2}} \neq 0$. Then $\bar{z} \in \operatorname{soc} \Lambda$, so $\bar{z}=\sum_{i=1}^{t} b_{i} \bar{p}_{i}$, with $b_{i} \in k$ and $p_{i} \in \mathbb{M}$ for all $i=1, \ldots, t$. Since $\bar{z} \neq 0$, there exists $j$ such that $b_{j} \neq 0$. Thus $\bar{p}_{j}^{*}(\bar{z}) \neq 0$. From this it follows that if $v$ is a path in $Q_{\Lambda}$ such that $\bar{v} \neq 0$, then $\beta_{p_{j}} \delta_{1} v \delta_{2}$ is an elementary cycle containing $v$.

Definition 3.4. Let $q$ be a path in an elementary cycle $\mathbb{C}$ of length less than or equal to the length of $\mathbb{C}$. If $s(q)=e(q)$, the supplement of $q$ in $\mathbb{C}$ is the trivial path $e_{s(q)}$; otherwise, it is the path formed by the remaining arrows of $\mathbb{C}$.

Consider the morphism of $k$-algebras $\Phi: k Q_{T(\Lambda)} \rightarrow T(\Lambda)$ defined on the trivial paths and the arrows as follows:

$$
\begin{aligned}
& \Phi\left(e_{i}\right)=\left(\overline{e_{i}}, 0\right), \\
& \text { for } i=1, \ldots, n \text {, } \\
& \Phi(\alpha)=(\bar{\alpha}, 0), \quad \Phi\left(\beta_{p}\right)=\left(0, \bar{p}^{*}\right), \quad \text { for } \alpha \in\left(Q_{\Lambda}\right)_{1} \text { and } p \in \mathbb{M} \text {. }
\end{aligned}
$$

Then $\Phi$ is surjective. Associated with $\Phi$ are the morphisms

$$
\varphi_{1}=\pi_{1} \Phi: k Q_{T(\Lambda)} \rightarrow \Lambda \text { and } \varphi_{2}=\pi_{2} \Phi: k Q_{T(\Lambda)} \rightarrow D(\Lambda),
$$

where $\pi_{1}, \pi_{2}$ are the projections induced by the decomposition $T(\Lambda)=$ $\Lambda \oplus D(\Lambda)$.

We now make some simple but important observations.

Throughout this section, $\left(\beta_{p}\right)_{p \in \mathbb{M}}$ denotes the ideal generated by the elements $\beta_{p}$ in $k Q_{T(\Lambda)}$.

LEMMA 3.5. Let $\Lambda=k Q_{\Lambda} /$ I be an algebra such that any oriented cycle in $k Q_{\Lambda}$ is zero in $\Lambda$ and $q$, $u$ be paths in $K Q_{T}$. $\left.\varphi_{2}\left(v_{2}\right)\right)$.

(a) If $v=v_{1}+v_{2}$, with $v_{1} \in k Q_{\Lambda}, v_{2} \in\left(\beta_{p}\right)_{p \in \mathbb{M}}$, then $\Phi(v)=\left(\varphi_{1}\left(v_{1}\right)\right.$,

(b) $\varphi_{2}(q) \neq 0$ implies that $q \in\left(\beta_{p}\right)_{p \in \mathbb{M}}$.

(c) $\varphi_{2}(q)=0$ if $q$ contains two or more arrows $\beta_{p}, p \in \mathbb{M}$.

(d) $\varphi_{2}(q)(\bar{u}) \neq 0$ implies that $u$ is a supplement of $q$. $k Q_{\Lambda}$.

(e) $\varphi_{2}(v)(\bar{u})=\varphi_{2}(v u)\left(\overline{e_{i}}\right)=\varphi_{2}(u v)\left(\overline{e_{j}}\right)$ if $u$ is a path from $i$ to $j$ in 
(f) If $v=\sum_{s=1}^{l} a_{s} q_{s}$, with $q_{s}$ different paths and $\varphi_{2}(v) \neq 0$, then there exists a supplement $u$ of one of the $q_{i}$ s such that $\varphi_{2}(v u) \neq 0$ and $\varphi_{2}(u v) \neq 0$.

(g) Let $\mathbb{C}$ be an elementary cycle with origin e. Then $\varphi_{2}(\mathbb{C})(\bar{e})=w(\mathbb{C})$ and $\varphi_{2}(\mathbb{C})(\bar{u})=0$ for any path $u$ in $k Q_{\Lambda}, u \neq e$.

(h) If $q$ has a supplement, then $\Phi(q) \neq 0$.

(i) Let $v \in e_{j} k Q_{T(\Lambda)} e_{i}$ and $\gamma$ be a path from $j$ to $i$. Then $v \gamma \in \operatorname{Ker} \Phi$ if and only if $\gamma v \in \operatorname{Ker} \Phi$.

Proof. (a), (b), and (e) follow directly from the definitions, while (g) follows from the definitions together with the hypothesis over $\Lambda$.

We get (c) using that $D(\Lambda)^{2}=0$ in $T(\Lambda)$. Assume $\varphi_{2}(q)(\bar{u}) \neq 0$. To prove (d), we know by (b) and (c) that $q=\gamma \beta_{p} \delta$, with $\gamma, \delta$ paths in $k Q_{\Lambda}$. Then $0 \neq \varphi_{2}(q)(\bar{u})=\bar{p}^{*}(\overline{\delta u \gamma})$, and therefore $u$ is a supplement of $q$ in the elementary cycle $\gamma \beta_{p} \delta u$.

Assume now $v$ as in (f) and let $u$ be such that $\varphi_{2}(v)(\bar{u}) \neq 0$. Hence $\varphi_{2}\left(q_{s}\right)(\bar{u}) \neq 0$ for some $s$; thus $u$ is a supplement of $q_{s}$, by (d). Now (f) follows from (e).

Suppose now that $u$ is a supplement for $q$ in $\mathbb{C}, \mathbb{C}=q u$. By $(\mathrm{g})$, $\varphi_{2}(\mathbb{C}) \neq 0$; thus $\Phi(q) \neq 0$.

Finally, let $v \in e_{j} k Q_{T(\Lambda)} e_{i}$ and let $\gamma$ be a path from $j$ to $i$. Then $\gamma v$ and $v \gamma$ are linear combinations of cycles with origin $i$ and $j$, respectively, which we may assume are in $\left(\beta_{p}\right)$ because $Q_{\Lambda}$ has no nonzero oriented cycles. Thus $\varphi_{2}(\gamma v), \varphi_{2}(v \gamma)$ vanish on all paths different from $e_{i}$ and $e_{j}$, respectively. By (e), we have $\varphi_{2}(\gamma v)\left(\overline{e_{i}}\right)=\varphi_{2}(v \gamma)\left(\overline{e_{j}}\right)$, and the statement follows now from (a), using the fact that $\gamma v$ and $v \gamma$ are in $\left(\beta_{p}\right)$.

In order to describe the relations for $T(\Lambda)$, we have to find generators for $\operatorname{Ker} \Phi$.

Proposition 3.6. Let $\Phi$ be as above. For each $j \in\left(Q_{T(\Lambda)}\right)_{0}$, let $I_{j}^{\prime}$ be the ideal in $k Q_{T(\Lambda)}$ generated by

(i) oriented cycles from $j$ to $j$ which are not elementary, and

(ii) elements $w\left(\mathbb{C}^{\prime}\right) \mathbb{C}-w(\mathbb{C}) \mathbb{C}^{\prime}$, where $\mathbb{C}, \mathbb{C}^{\prime}$ are elementary cycles with origin $j$.

Then $\operatorname{Ker} \Phi \cap e_{j} k Q_{T(\Lambda)} e_{j}$ generates $I_{j}^{\prime}$.

Proof. That oriented cycles in $k Q_{\Lambda}$ lie in $\operatorname{Ker} \Phi$ is a direct consequence of the definition of $\Phi$ and the hypothesis on $\Lambda$. So let $\mathbb{C}$ be a cycle from $j$ to $j$ in $k Q_{T(\Lambda)}$, that is, in $\left(\beta_{p}\right)$. Then $\Phi(\mathbb{C})=\left(0, \varphi_{2}(\mathbb{C})\right)$, by Lemma 3.5(a). If $\mathbb{C}$ contains two or more arrows $\beta_{p}, p \in \mathbb{M}$, then $\varphi_{2}(\mathbb{C})=0$ by (c) of the same lemma, and therefore $\mathbb{C}$ lies in $\operatorname{Ker} \Phi$. Suppose now that $\mathbb{C}$ contains exactly one arrow $\beta_{p}$, for some $p \in \mathbb{M}$. Then $\mathbb{C}=\gamma \beta_{p} \delta$, with $\gamma, \delta$ paths in $k Q_{\Lambda}$. If $\varphi_{2}(\mathbb{C}) \neq 0$, there exists a path $u$ in $k Q_{\Lambda}$ such that 
$\varphi_{2}(\mathbb{C})(u)=\bar{p}^{*}(\overline{\delta u \gamma}) \neq 0$. Hence $u$ is a path from $j$ to $j$ and $\bar{u} \neq 0$. It follows from the hypothesis on $\Lambda$ that $u=e_{j}$, so $\mathbb{C}$ is elementary. Thus nonelementary cycles are in $\operatorname{Ker} \Phi$.

Let now $z=w\left(\mathbb{C}^{\prime}\right) \mathbb{C}-w(\mathbb{C}) \mathbb{C}^{\prime}$ be an element as defined in (ii). Then $\Phi(z)=\left(0, \varphi_{2}(z)\right)$, and $\varphi_{2}(z)=w\left(\mathbb{C}^{\prime}\right) \varphi_{2}(\mathbb{C})-w(\mathbb{C}) \varphi_{2}\left(\mathbb{C}^{\prime}\right)$.

Let $u$ be a path in $k Q_{\Lambda}$. By Lemma $3.5(\mathrm{~g})$, we have that $\varphi_{2}(z)(\bar{u})=0$ if $u \neq e_{j}$, and $\varphi_{2}(\mathbb{C})\left(\overline{e_{j}}\right)=w(\mathbb{C}), \varphi_{2}\left(\mathbb{C}^{\prime}\right)\left(\overline{e_{j}}\right)=w\left(\mathbb{C}^{\prime}\right)$, so $\varphi_{2}(z)\left(\overline{e_{j}}\right)=0$.

Thus $I_{j}^{\prime}$ is contained in the ideal generated by $\operatorname{Ker} \Phi \cap e_{j} k Q_{T(\Lambda)} e_{j}$. To prove the other inclusion, let $z \in \operatorname{Ker} \Phi \cap e_{j} k Q_{T(\Lambda)} e_{j}$. We write $z=z_{1}+z_{2}$, with $z_{1}=\sum_{i=1}^{r} a_{i} \mathbb{C}_{i}, z_{2}=\sum_{i=r+1}^{t} a_{i} \mathbb{C}_{i}$, where $\mathbb{C}_{1}, \ldots, \mathbb{C}_{r}$ are elementary cycles and $\mathbb{C}_{r+1}, \ldots, \mathbb{C}_{t}$ are cycles which are not elementary.

Then $z_{2} \in I_{j}^{\prime}$, and we just proved that $I_{j}^{\prime} \subseteq \operatorname{Ker} \Phi$. So $0=\Phi(z)=\Phi\left(z_{1}\right)$. Since $\mathbb{C}_{i}$ is elementary, $\mathbb{C}_{i} \in\left(\beta_{p}\right)$, so $\Phi\left(\mathbb{C}_{i}\right)=\left(0, \varphi_{2}\left(\mathbb{C}_{i}\right)\right), i=1, \ldots, r$. Thus $0=\Phi\left(z_{1}\right)=\left(0, \sum_{i=1}^{r} a_{i} \varphi_{2}\left(\mathbb{C}_{i}\right)\right)$, and we get from Lemma $3.5(\mathrm{~g})$

$$
0=\sum_{i=1}^{r} a_{i} \varphi_{2}\left(\mathbb{C}_{i}\right)\left(\bar{e}_{j}\right)=\sum_{i=1}^{r} a_{i} w\left(\mathbb{C}_{i}\right)
$$

So $a_{1}=-\sum_{i=2}^{r} a_{i} w\left(\mathbb{C}_{i}\right) / w\left(\mathbb{C}_{1}\right)$, and $z_{1}=\sum_{i=2}^{r}\left(a_{i} \mathbb{C}_{i}-a_{i}\left(w\left(\mathbb{C}_{i}\right) /\right.\right.$ $\left.\left.w\left(\mathbb{C}_{1}\right)\right) \mathbb{C}_{1}\right)=\sum_{i=2}^{r}\left(a_{i} / w\left(\mathbb{C}_{1}\right)\right)\left(\left(w\left(\mathbb{C}_{1}\right) \mathbb{C}_{i}-w\left(\mathbb{C}_{i}\right) \mathbb{C}_{1}\right)\right) \in I_{j}^{\prime}$, since it is a linear combination of elements of the type (ii). Thus the ideal generated by $\operatorname{Ker} \Phi \cap e_{j} k Q_{T(\Lambda)} e_{j}$ is contained in $I_{j}^{\prime}$, and therefore coincides with $I_{j}^{\prime}$.

Remark 3.7. As a direct consequence of the preceding proposition, we know that the classes of oriented cycles with origin $j$ in $k Q_{T(\Lambda)}$ generate a one-dimensional subspace of $k Q_{T(\Lambda)} / I_{j}^{\prime}$.

We have now the following consequence of Proposition 3.6.

Corollary 3.8. Let $\Lambda$ be as in Lemma 3.5 and let $\mathbb{C}$ be an oriented cycle in $Q_{T(\Lambda)}$. Then the following conditions are equivalent:

(i) $\mathbb{C}$ is an elementary cycle.

(ii) $\mathbb{C}$ is nonzero in $T(\Lambda)$.

Proof. It follows from Lemma 3.5(g) that (i) implies (ii).

We get directly from Proposition 3.6 that (ii) implies (i), since nonelementary cycles are in $I_{j}^{\prime}$, which is contained in $\operatorname{Ker} \Phi$.

We can now prove the main result of this section.

THEOREM 3.9. Let $\Lambda=k Q_{\Lambda} /$ I be an algebra such that any oriented cycle in $Q_{\Lambda}$ is zero in $\Lambda$. Let $I^{\prime}$ be the ideal in $k Q_{T(\Lambda)}$ generated by

(i) $I$,

(ii) the paths consisting of $n+1$ arrows of an elementary cycle of length $n$, 
and

(iii) the paths whose arrows do not belong to a single elementary cycle,

(iv) the elements $\sum_{s=1}^{l} a_{s} \mu_{s}$, where $a_{s} \in k^{*}$ and $\mu_{s}$ are different paths from $i$ to $j$ in $\left(\beta_{p}\right)_{p \in \mathbb{M}}$, for $s=1, \ldots, l$, and such that

$$
\gamma\left(\sum_{s=1}^{l} a_{s} \mu_{s}\right) \in I_{i}^{\prime} \quad \text { or } \quad\left(\sum_{s=1}^{l} a_{s} \mu_{s}\right) \gamma \in I_{j}^{\prime},
$$

where $I_{t}^{\prime}$ is the ideal defined in Proposition 3.6, for each supplement $\gamma$ of one of the $\mu_{s} s$.

Then $I^{\prime}$ is admissible and $I^{\prime}=I_{T(\Lambda)}$. That is, $T(\Lambda) \simeq k Q_{T(\Lambda)} / I^{\prime}$.

Proof. It is sufficient to prove that $I^{\prime}=\operatorname{Ker} \Phi$, where $\Phi$ is the morphism defined above. First we show that $I^{\prime} \subseteq \operatorname{Ker} \Phi$.

Let $q$ be a path in $k Q_{T(\Lambda)}$, which is not in $\operatorname{Ker} \Phi$. If $\varphi_{2}(q) \neq 0$, then $q=\gamma \beta_{p} \delta$, with $p \in \mathbb{M}$ and $\gamma, \delta$ paths in $k Q_{\Lambda}$. Let $u$ in $k Q_{\Lambda}$ be a path such that $\varphi_{2}(q)(\bar{u}) \neq 0$. Then we have $0 \neq \bar{p}^{*}(\overline{\delta u \gamma})=\varphi_{2}(q)(\bar{u})$.

Since $\Lambda$ has no nonzero oriented cycles, $\delta$ and $\gamma$ contain no common arrows. Thus $q$ cannot consist of $n+1$ arrows in an elementary cycle of length $n$. Moreover, $q$ is in the elementary cycle $\mathbb{C}=q u$.

If $\varphi_{1}(q) \neq 0$, then $q$ is a path in $k Q_{\Lambda}$, and clearly $q \notin I$. We know that $q$ is contained in an elementary cycle, by Remark 3.3. So, if $I^{\prime \prime}$ is the ideal generated by the classes (i)-(iii) of $k Q_{T(\Lambda)}$, then $q \notin I^{\prime \prime}$, and so $I^{\prime \prime} \subseteq \operatorname{Ker} \Phi$.

Assume now that $v=\sum_{s=1}^{l} a_{s} q_{s} \notin \operatorname{Ker} \Phi$, where the $q_{s}$ s are different paths in $\left(\beta_{p}\right)_{p \in \mathbb{M}}$ from $i$ to $j$. Then $\Phi(v)=\left(0, \varphi_{2}(v)\right) \neq 0$.

Recall now that we know from Proposition 3.6 that $I_{j}^{\prime} \subseteq \operatorname{Ker} \Phi$ for all $j$. Then, if $\varphi_{2}(v) \neq 0$, Lemma 3.5(f) states precisely that $v$ is not an element in the class (iv).

Thus we have shown that $q \notin \operatorname{Ker} \Phi$ implies $q \notin I^{\prime}$. This completes the proof that $I^{\prime} \subseteq \operatorname{Ker} \Phi$.

Since $\Phi: k Q_{T(\Lambda)} \rightarrow T(\Lambda)$ is surjective and $I^{\prime} \subseteq \operatorname{Ker} \Phi$, to prove that the equality holds it is enough to prove that $\operatorname{dim}_{k} k Q_{T(\Lambda)} / I^{\prime}=\operatorname{dim}_{k} T(\Lambda)=$ $2 \operatorname{dim}_{k} \Lambda$.

The image of an element $y \in k Q_{T(\Lambda)}$ under the canonical epimorphism $k Q_{T(\Lambda)} \rightarrow k Q_{T(\Lambda)} / I^{\prime}$ is denoted by $\tilde{y}$.

Since $I^{\prime} \subseteq \operatorname{Ker} \Phi$, we have the canonical epimorphism $k Q_{T(\Lambda)} / I^{\prime} \rightarrow$ $k Q_{T(\Lambda)} / \operatorname{Ker} \Phi=T(\Lambda)$. The inclusion of $\Lambda$ in $T(\Lambda)$ factors through $k Q_{T(\Lambda)} / I^{\prime}$ because $I \subseteq I^{\prime}$. Thus the map $\iota: \Lambda \rightarrow k Q_{T(\Lambda)} / I^{\prime}$ induced by the embedding of $k Q_{\Lambda}$ in $k Q_{T(\Lambda)}$ is a monomorphism.

We have that $k Q_{T(\Lambda)}=k Q_{\Lambda}+\left(\beta_{p}\right)_{p \in \mathbb{M}}$. Therefore, $e_{j} k Q_{T(\Lambda)} e_{i}=$ $e_{j} k Q_{\Lambda} e_{i}+e_{j}\left(\beta_{p}\right)_{p \in \mathbb{M}} e_{i}$, for each $i$ and $j$ in $\left(Q_{T(\Lambda)}\right)_{0}$. Let $\pi: k Q_{T(\Lambda)} \rightarrow$ $k Q_{T(\Lambda)} / I^{\prime}$ be the canonical epimorphism. 
We define in $k Q_{T(\Lambda)} / I^{\prime}$ the subspaces $\mathscr{P}_{i j}=\pi\left(e_{j} k Q_{\Lambda} e_{i}\right)$ and $\mathscr{F}_{i j}=$ $\pi\left(e_{j}\left(\beta_{p}\right)_{p \in \mathbb{M}} e_{i}\right)$. Then $\mathscr{P}_{i j}=\iota\left(e_{j} \Lambda e_{i}\right) \simeq e_{j} \Lambda e_{i}$, so $\sum_{i, j} \operatorname{dim}_{k} \mathscr{P}_{i j}=\operatorname{dim}_{k} \Lambda$.

We will prove that $\operatorname{dim}_{k}\left(\mathscr{P}_{i j}\right) \geq \operatorname{dim}_{k}\left(\mathscr{F}_{j i}\right)$. Though these dimensions depend on $i$ and $j$, to simplify notation we will denote them by $n$ and $m$, respectively.

We observe first that a path $q$ in $k Q_{T(\Lambda)}$ is not in $I^{\prime}$ if and only if there is a supplement for $q$ in some elementary cycle $\mathbb{C}$. In fact, we know by Corollary 3.8 that elementary cycles are nonzero in $T(\Lambda)$ and therefore are not in $I^{\prime}$, because $I^{\prime} \subseteq \operatorname{Ker} \Phi$. On the other hand, if $q \notin I^{\prime}$, then all arrows of $q$ belong to a single elementary cycle $\mathbb{C}$ since $q$ does not belong to the class (iii) of $I^{\prime}$. Moreover, using the fact that $q$ is not in the class (ii) of $I^{\prime}$, we deduce that $q$ is contained in $\mathbb{C}$.

As a first consequence, we find that $\mathscr{F}_{j i} \neq 0$ if and only if $\mathscr{P}_{i j} \neq 0$. So we assume that both are nonzero and choose paths $\gamma_{1}, \ldots, \gamma_{n}$ in $k Q_{\Lambda}$ and $\mu_{1}, \ldots, \mu_{m}$ in $\left(\beta_{p}\right)_{p \in \mathbb{M}}$, so that $\left\{\tilde{\gamma_{1}}, \ldots, \widetilde{\gamma_{n}}\right\}$ and $\left\{\widetilde{\mu_{1}}, \ldots, \widetilde{\mu_{m}}\right\}$ are bases for $\mathscr{P}_{i j}$ and $\mathscr{F}_{j i}$, respectively.

Now we will show that $m \leq n$. Suppose on the contrary that $m>n$. We will find a nonzero element $w$ in $\mathscr{F}_{j i}$ such that $w \tilde{\gamma}=0$ for each supplement $\gamma$ of any of the $\mu_{t}$. This will contradict that $w$ does not belong to the class (iv) of $I^{\prime}$. Clearly, it is enough to find $w \neq 0$ in $\mathscr{F}_{j i}$ such that $w \tilde{\gamma}_{t}=0$ for each $t$. So we start looking for relations among the $\mu_{k} \gamma_{t}$ s.

Let $1 \leq t \leq n$. We prove first that not all $\widehat{\mu_{k} \gamma_{t}}, k=1, \ldots, m$, are zero. In fact, $\gamma_{t} \notin I^{\prime}$, so, as we observed above, it has a supplement, say $\delta_{t}$, in an elementary cycle $\mathbb{C}_{t}$. Using again the above observation, we get that $\delta_{t} \gamma_{t} \notin I^{\prime}$. Now, $\tilde{\delta}_{t} \in \mathscr{F}_{j i}$ because $\tilde{\gamma}_{t} \in \mathscr{P}_{i j}$, so $\tilde{\delta}_{t}$ is a linear combination of $\widetilde{\mu_{1}}, \ldots, \widetilde{\mu_{m}}$, and therefore, for every $t$ with $1 \leq t \leq n$, there exists an index $r_{t}$ such that $\widetilde{\mu_{r_{t}} \gamma_{t}} \neq 0$ and $1 \leq r_{t} \leq m$. Now we know from Remark 3.7 that the cycles $\widetilde{\mu_{k} \gamma_{t}}, k=1, \ldots, m$, generate a subspace of $k Q_{T(\Lambda)} / I^{\prime}$ of dimension 1 , because all these cycles have origin $i$. We conclude that $\widetilde{\mu_{k}} \gamma_{t}=$ $a_{k t} \widetilde{\mu_{r_{t}} \gamma_{t}}$ for some $a_{k t} \in k$ and for all $k=1, \ldots, m$.

Now, $\left(\sum_{k=1}^{m} x_{k} \widetilde{\mu_{k}}\right) \widetilde{\gamma}_{t}=\sum_{k=1}^{m} x_{k} a_{k t} \widetilde{\mu_{r_{t}} \gamma_{t}}$. The system $\sum_{k=1}^{m} a_{k t} X_{k}=0$, with $t=1, \ldots, n$, has a nontrivial solution because $m>n$, say $\left(x_{1}, \ldots, x_{m}\right)$. Then the element $w=\sum_{k=1}^{m} x_{k} \widetilde{\mu_{k}}$ satisfies the required conditions: $w \neq 0$ and $w \tilde{\gamma}_{t}=0$ for all $t$, ending the proof that $m \leq n$.

We are now in a position to prove that $\operatorname{dim}_{k} k Q_{T(\Lambda)} / I^{\prime}=\operatorname{dim}_{k} T(\Lambda)$. Since $k Q_{T(\Lambda)} / I^{\prime}$ maps onto $T(\Lambda)$, we get that $\operatorname{dim}_{k} k Q_{T(\Lambda)} / I^{\prime} \geq \operatorname{dim}_{k} T(\Lambda)$. On the other hand, $\operatorname{dim}_{k} k Q_{T(\Lambda)} / I^{\prime} \leq \sum_{i, j}\left(\operatorname{dim}_{k} \mathscr{P}_{i j}+\operatorname{dim}_{k} \mathscr{F}_{i j}\right) \leq$ $\sum_{i, j}\left(\operatorname{dim}_{k} \mathscr{P}_{i j}+\operatorname{dim}_{k} \mathscr{P}_{j i}\right)=2 \operatorname{dim}_{k} \Lambda=\operatorname{dim} T(\Lambda)$. This ends the proof of the theorem.

As an immediate consequence, we get that any nonzero path in $T(\Lambda)$ is in a nonzero oriented cycle. 
We will see next that the hypothesis that $\mu_{s} \in\left(\beta_{p}\right)_{p \in \mathbb{M}}$ in (iv) of the preceding theorem can be omitted.

COROLlARY 3.10. Let $v=\sum_{s=1}^{l} a_{s} q_{s}$, where $a_{s} \in k^{*}$ and the $q_{s}$ are pairwise different paths from $i$ to $j$ in $k Q_{T(\Lambda)}$. Suppose that either $\overline{\gamma v}=0$ or $\overline{v \gamma}=0$ in $T(\Lambda)$ for every supplement $\gamma$ of any of the $q_{s}$ s. Then $\bar{v}=0$.

Proof. Suppose first that $v=\sum_{s=1}^{l} a_{s} q_{s}$ satisfies $\overline{\gamma v}=0$ for a supplement $\gamma$ of some $q_{s}$ s. Then, for some $0 \leq r \leq l$, we may suppose that $q_{1}, \ldots, q_{r} \in$ $k Q_{\Lambda}, q_{r+1}, \ldots, q_{l} \in\left(\beta_{p}\right)_{p \in \mathbb{M}}$. Let $v_{1}=\sum_{s=1}^{r} a_{s} q_{s}, v_{2}=\sum_{s=r+1}^{l} a_{s} q_{s}$.

Assume that $\gamma$ is a supplement of $q_{j}$, with $j>r$. Then $\gamma \in k Q_{\Lambda}$, and therefore $\overline{\gamma v_{1}}=0$ because oriented cycles are zero in $\Lambda$. Thus $\overline{\gamma v_{2}}=0$ and we conclude from the preceding theorem that $\overline{v_{2}}=0$.

So $v=v_{1} \in k Q_{\Lambda}$. If $\bar{v}_{1} \neq 0$, then we know by Remark 3.3 that there exist $\delta_{1}, \delta_{2} \in k Q_{\Lambda}$ and $p \in \mathbb{M}$ so that $\bar{p}^{*}\left(\overline{\delta_{1} v \delta_{2}}\right) \neq 0$, and thus, for some $s, \bar{p}^{*}\left(\overline{\delta_{1} q_{s} \delta_{2}}\right) \neq 0$, so $\mathbb{C}=\beta_{p} \delta_{1} q_{s} \delta_{2}$ is an elementary cycle. Then $\gamma=$ $\delta_{2} \beta_{p} \delta_{1}$ is a supplement of $q_{s}$. Moreover,

$$
\varphi_{2}(\gamma)(\bar{v})=\left(\overline{\delta_{2}} \bar{p}^{*} \overline{\delta_{1}}\right)(\bar{v})=\bar{p}^{*}\left(\overline{\delta_{1} v \delta_{2}}\right) \neq 0 .
$$

We conclude from Lemma 3.5(e) that $\varphi_{2}(\gamma v) \neq 0$. So $\Phi(\gamma v) \neq 0$, contradicting the assumption that $\overline{\gamma v}=0$ in $k Q_{T(\Lambda)}$. Thus we have $\bar{v}=0$, as desired. This ends the proof of the corollary, since we know by Lemma 3.5(i) that $\overline{v \gamma}=0$ if and only if $\overline{\gamma v}=0$.

When the algebra $\Lambda$ is schurian, the description of the ideal $I^{\prime}$ is easier, as we state in the following corollary.

Corollary 3.11. Let $\Lambda=k Q_{\Lambda} / I$ be a schurian algebra. The ideal $I^{\prime \prime}$ generated by

(a) the paths consisting of $n+1$ arrows of an elementary cycle of length $n$, and

(b) the paths whose arrows do not belong to a single elementary cycle,

(c) the elements $a^{\prime} q-a q^{\prime}$, where $q, q^{\prime}$ are paths from $i$ to $j$ admitting a common supplement $\gamma \in k Q_{\Lambda}$ in elementary cycles,

$$
\beta_{p} \alpha_{n} \cdots \alpha_{1} \text { and } \beta_{p^{\prime}} \alpha_{m}^{\prime} \cdots \alpha_{1}^{\prime} \text {, }
$$

respectively, with $\overline{\alpha_{n} \cdots \alpha_{1}}=a \bar{p}$ and $\overline{\alpha_{m}^{\prime} \cdots \alpha_{1}^{\prime}}=a^{\prime} \overline{p^{\prime}}, a, a^{\prime} \in k^{*}$,

is admissible and $I^{\prime \prime}=I_{T(\Lambda)}$. That is, $T(\Lambda) \simeq k Q_{T(\Lambda)} / I^{\prime \prime}$.

Proof. First we observe, for a path $\gamma \in k Q_{\Lambda}$ and $p \in \mathbb{M}$, that $\bar{\gamma}=a \bar{p}$ with $a \in k$ if and only if $w\left(\beta_{p} \gamma\right)=a$. So the elements $\omega\left(\mathbb{C}^{\prime}\right) \mathbb{C}-\omega(\mathbb{C}) \mathbb{C}^{\prime}$ with $\mathbb{C}, \mathbb{C}^{\prime}$ cycles with the same origin are in the class (c) of $I^{\prime \prime}$. 
Assume now that $v=\sum_{s=1}^{l} a_{s} \mu_{s}$ is an element in the class (iv) of $I^{\prime}=$ $I_{T(\Lambda)}$, where $a_{s} \in k^{*}$ and $\mu_{s}$ are different paths from $i$ to $j$ in $\left(\beta_{p}\right)_{p \in \mathbb{M}}$, for $s=1, \ldots, l$. That is, $\overline{\gamma v} \in I_{i}^{\prime}$ for any supplement $\gamma$ of one of the $\mu_{s}$ s. We may assume that $\mu_{1}, \ldots, \mu_{l}$ are not in the class (b) of $I^{\prime \prime}$. Since $\Lambda$ is schurian, they have the same supplements. Let $\gamma$ be one of them.

Then $\overline{\gamma \mu_{i}}=\left(\omega\left(\overline{\gamma \mu_{i}}\right) / \omega\left(\overline{\gamma \mu_{1}}\right)\right) \overline{\gamma \mu_{1}}$ by Proposition 3.6. So

$$
0=\overline{\gamma v}=\left(a_{1}+\sum_{i=2}^{l} a_{i} \omega\left(\overline{\gamma \mu_{i}}\right) / \omega\left(\overline{\gamma \mu_{1}}\right)\right) \overline{\gamma \mu_{1}} .
$$

Thus $a_{1}=-\sum_{i=2}^{l} a_{i} \omega\left(\overline{\gamma \mu_{i}}\right) / \omega\left(\overline{\gamma \mu_{1}}\right)$. By replacing this expression in $v$, we obtain that

$$
v=\sum_{i=2}^{l} a_{i}\left(\overline{\mu_{i}}-\left(\omega\left(\overline{\gamma \mu_{i}}\right) / \omega\left(\overline{\gamma \mu_{1}}\right)\right) \overline{\mu_{1}}\right)
$$

belongs to the class (c) of $I^{\prime \prime}$.

Now we will prove that $I \subseteq I^{\prime \prime}$. If a path $\gamma$ is in $I$, then $\gamma$ is in the class (b) of $I^{\prime \prime}$. If $\gamma, \gamma^{\prime}$ are paths in $k Q_{\Lambda}$ such that $\bar{\gamma}, \overline{\gamma^{\prime}} \neq 0$ in $\Lambda$ and $\gamma-a \gamma^{\prime} \in I$, then they have the same supplements, and we know by Corollary 3.8 that they have at least one. Let $\mu$ be one of them, and $\mathbb{C}=\mu \gamma$ and $\mathbb{C}^{\prime}=\mu \gamma^{\prime}$. Then $\Phi(\mathbb{C})=a \Phi\left(\mathbb{C}^{\prime}\right)$, so $w(\mathbb{C})=a w\left(\mathbb{C}^{\prime}\right)$ and thus $\gamma-a \gamma^{\prime}$ is in the class (iv) of $I^{\prime}$ and therefore in $I^{\prime \prime}$. So $I \subseteq I^{\prime \prime}$.

Since the classes (a), (b) of $I^{\prime \prime}$ coincide with the classes (ii), (iii) of $I^{\prime}$, we have that $I^{\prime} \subseteq I^{\prime \prime}$.

On the other hand, the previous corollary shows that the class (c) of $I^{\prime \prime}$ is contained in $I^{\prime}$, proving that $I^{\prime \prime} \subseteq I^{\prime}$.

Suppose $\Lambda$ is a schurian algebra such that the ordinary quiver of $\Lambda$ has no oriented cycles. Then all oriented cycles in $k Q_{T(\Lambda)}$ not passing through any vertex more than once are nonzero in $T(\Lambda)$. So we can replace "elementary cycle" by "cycle" in the statement of Corollary 3.11 .

Schurian algebras have the property that if $\gamma$ and $\eta$ are paths in $k Q_{\Lambda}$ from $i$ to $j$ and $\bar{\gamma}, \bar{\eta} \neq 0$ in $\Lambda$, then $\bar{\gamma}=a \bar{\eta}$, with $a \in k$. Sometimes the presentation for $\Lambda$ can be chosen so that always $a=1$, and we say then that parallel paths are equal in $\Lambda$. In this case, the class (c) in Corollary 3.11 can be described in a simpler way, and if we further assume that the ordinary quiver of $\Lambda$ has no oriented cycles, then the situation is particularly nice, because the relations can be formulated directly in terms of the cycles in $k Q_{T(\Lambda)}$, independently of the relations for $\Lambda$, as we state in the following corollary.

Corollary 3.12. Let $\Lambda=k Q_{\Lambda} / I$ be a schurian algebra such that the ordinary quiver of $\Lambda$ has no oriented cycles and parallel paths in $Q_{\Lambda}$ are equal in $\Lambda$. Then the ideal $I_{T(\Lambda)}$ of relations for $T(\Lambda)$ is generated by

(i) the paths consisting of $n+1$ arrows in a cycle of length $n$, 
(ii) the paths whose arrows do not belong to a single cycle, and

(iii) the difference $q-q^{\prime}$ of paths $q, q^{\prime}$ with the same origin and endpoint and having a common supplement in cycles of $Q_{T(\Lambda)}$.

We observe here that the hypothesis of Corollary 3.12 holds for any algebra $\Lambda$ such that $T(\Lambda)$ is of finite representation type [Y].

Now we give two examples.

EXAMPLE 3.13. Let $\Lambda$ be as in Example 2.5. Using the description of $k Q_{T(\Lambda)}$ given there and Corollary 3.12, we find that $T(\Lambda)$ is the algebra given by $k Q_{T(\Lambda)}$ with the relations

$$
\begin{aligned}
\mathfrak{r}^{4} & =0, \\
\alpha_{1} \beta_{p_{1}} & =\beta_{p_{2}} \alpha_{3}, \\
\alpha_{3} \alpha_{2} \alpha_{1} & =\beta_{p_{1}} \alpha_{2} \beta_{p_{2}}=0 .
\end{aligned}
$$

EXAMPLE 3.14. Let $\Lambda$ be as in Example 2.6. This algebra is not schurian. In this case we find that $T(\Lambda)$ is the algebra given by $Q_{T(\Lambda)}$ with the relations

$$
\begin{aligned}
\alpha_{3} \alpha_{1} & =\alpha_{3} \alpha_{2} ; \quad \alpha_{4} \alpha_{1}=\alpha_{4} \alpha_{2}, \\
\alpha_{1} \beta_{p_{2}} \alpha_{3} \alpha_{1} & =\alpha_{3} \alpha_{1} \beta_{p_{2}} \alpha_{3}=\beta p_{2} \alpha_{3} \alpha_{1} \beta p_{2}=0, \\
\alpha_{4} \alpha_{1} \beta_{p_{3}} \alpha_{4} & =\beta_{p_{3}} \alpha_{4} \alpha_{1} \beta_{p_{3}}=0 \\
\alpha_{2} \beta_{p_{1}} \alpha_{2} & =\beta_{p_{1}} \alpha_{2} \beta_{p_{1}}=0 \\
\alpha_{3} \alpha_{1} \beta_{p_{3}} & =0 ; \quad \beta_{p_{1}} \alpha_{1}=\alpha_{1} \beta_{p_{1}}=0 ; \quad \beta_{p_{1}} \alpha_{1} \beta_{p_{3}}=0, \\
\alpha_{4} \alpha_{1} \beta_{p_{2}} & =0 ; \quad \beta_{p_{1}} \alpha_{2} \beta_{p_{2}}=0, \\
\beta_{p_{2}} \alpha_{3} & =\beta_{p_{3}} \alpha_{4} ; \quad \alpha_{1} \beta_{p_{2}}=\alpha_{2} \beta_{p_{2}} ; \quad \alpha_{1} \beta_{p_{3}}=\alpha_{2} \beta_{p_{3}} ; \quad \beta_{p_{1}} \alpha_{2}=\beta_{p_{2}} \alpha_{3} \alpha_{1} .
\end{aligned}
$$

We go on now to study the ordinary quiver and relations for the repetitive algebra $\widehat{\Lambda}$ of $\Lambda$, as defined by Hughes and Waschbusch in [HW],

$$
\widehat{\Lambda}=\left(\begin{array}{ccccc}
\ddots & \ddots & & & 0 \\
& \Lambda_{m-1} & Q_{m-1} & & \\
& & \Lambda_{m} & Q_{m} & \\
& & & \Lambda_{m+1} & \ddots \\
0 & & & & \ddots
\end{array}\right),
$$

where $\Lambda_{m}=\Lambda, Q_{m}=D(\Lambda)$ for all $m \in Z$. 
The vertices of $Q_{\widehat{\Lambda}}$ are pairs $(m, i)$ with $m \in Z, i \in\left(Q_{\Lambda}\right)_{0}$, and the set of arrows is

$$
\begin{aligned}
\left(Q_{\widehat{\Lambda}}\right)_{1}= & \left\{\alpha^{m}:(m, i) \rightarrow(m, j)\right\}_{\alpha: i \rightarrow j \in\left(Q_{\Lambda}\right)_{1}} \\
& \cup\left\{\beta_{p}^{m}:(m, e(p)) \rightarrow(m+1, s(p))\right\}_{p \in \mathbb{M}}
\end{aligned}
$$

for $m \in Z$.

It is well known that $\widehat{\Lambda}$ is a Galois covering of $T(\Lambda)$. If $\gamma=\alpha_{1} \cdots \alpha_{n}$ is a path in $k Q_{\Lambda}$, we will denote by $\gamma^{m}$ the path $\alpha_{1}^{m} \cdots \alpha_{n}^{m}$ in $k Q_{\widehat{\Lambda}}$.

The relations are obtained by lifting the relations in $k Q_{T(\Lambda)}$. We have to replace the notions of elementary cycle, weight of an elementary cycle, and supplement of a path by the following notions.

Definition 3.15. A path $C$ in $k Q_{\widehat{\Lambda}}$ is called elementary if $C=$ $\delta^{m+1} \beta_{p}^{m} \gamma^{m}$, with $\delta, \gamma$ paths in $k Q_{\Lambda}, p \in \mathbb{M}$, such that $\bar{p}^{*}(\overline{\gamma \delta}) \neq 0$. In this case the weight of $C$ is $w(C)=p^{*}(\overline{\gamma \delta})$. A path $q$ in $k Q_{\widehat{\Lambda}}$ has a supplement if there is a path $q^{\prime}$ in $k Q_{\widehat{\Lambda}}$ such that $q q^{\prime}$ is an elementary path.

We are now in a position to describe the relations in $k Q_{\widehat{\Lambda}}$ generalizing a result by Asashiba for triangular schurian algebras such that parallel paths in $Q_{\Lambda}$ are equal in $\Lambda$ [A]. We start with the ideal $I_{\widehat{\Lambda}(m, j)}$ of relations from $(m, j)$ to $(m+1, j)$. This ideal is generated by

(a) paths from $(m, j)$ to $(m+1, j)$ which are not elementary,

(b) elements $w\left(C^{\prime}\right) C-w(C) C^{\prime}$, where $C, C^{\prime}$ are elementary paths from $(m, j)$ to $(m+1, j)$.

THEOREM 3.16. Let $\Lambda=k Q_{\Lambda} /$ I be an algebra such that any oriented cycle in $Q_{\Lambda}$ is zero in $\Lambda$. Let $I^{\prime}$ be the ideal in $k Q_{\widehat{\Lambda}}$ generated by

(a) the sets $I^{m}=\left\{\sum a_{i} \gamma_{i}^{m}\right.$, such that $\left.\sum a_{i} \gamma_{i} \in I\right\}, m \in Z$,

(b) the paths not contained in an elementary path, and

(c) the elements $x=\sum_{s=1}^{l} a_{s} q_{s}$, where $a_{s} \in k^{*}$ and $q_{s}$ are different paths from $(m, i)$ to $\left(m^{\prime}, j\right)$ in $\left(\beta_{p}^{m}\right)_{m \in Z, p \in \mathbb{M}}$ for $s=1, \ldots, l$, and such that

$$
\gamma x \in I_{\widehat{\Lambda}(m, i)} \text { and } \quad x \gamma \in I_{\widehat{\Lambda}\left(m^{\prime}, j\right)}
$$

for each supplement $\gamma$ of one of the $q_{s} s$ and $I_{\widehat{\Lambda}(m, i)}$ the above defined ideal.

Then $I^{\prime}$ is admissible and $I^{\prime}=I_{\widehat{\Lambda}}$. That is, $\widehat{\Lambda} \simeq k Q_{\widehat{\Lambda}} / I^{\prime}$.

Remark 3.17. We observe that the elements corresponding to the class (ii) of Theorem 3.9 belong to the class (b). 
Proof. Let $1_{\Lambda}^{m}$ be the element of $\widehat{\Lambda}$ which has $1_{\Lambda}$ as its $m$ th diagonal entry and all other entries zero, and let $e_{(m, i)}=1_{\Lambda}^{m} e_{i}$ be the idempotent of $\widehat{\Lambda}$ corresponding to the vertex $(m, i)$ of $Q_{\widehat{\Lambda}}$. We define $\Psi: k Q_{\widehat{\Lambda}} \rightarrow \widehat{\Lambda}$ by $\Psi((m, j))=e_{(m, j)}, \Psi\left(\alpha^{m}\right)=\bar{\alpha} \cdot 1_{\Lambda}^{m}$, and $\Psi\left(\beta_{p}^{m}\right)={\overline{p^{*}}}_{(m, m+1)}$, the matrix with $\overline{p^{*}}$ in the $(m, m+1)$ entry and zero elsewhere, for $p \in \mathbb{M}, m \in Z$.

Then $\Psi$ is surjective and $I^{\prime} \subseteq \operatorname{Ker} \Psi$. This can be proven with a straightforward adaptation of the arguments used in the proof of Theorem 3.9 (see also $[R])$. The other inclusion follows from the fact that

$$
\begin{aligned}
& \operatorname{dim}_{k} e_{(m, i)}\left(k Q_{\widehat{\Lambda}} / I^{\prime}\right) e_{(m, j)}+\operatorname{dim}_{k} e_{(m, i)}\left(k Q_{\widehat{\Lambda}} / I^{\prime}\right) e_{(m+1, j)} \\
& \quad=\operatorname{dim}_{k} e_{i}\left(k Q_{T(\Lambda)} / I_{T(\Lambda)}\right) e_{j} .
\end{aligned}
$$

Summing up over $i$ and $j$, we see that

$$
\operatorname{dim}_{k} 1_{\Lambda}^{m} \cdot k Q_{\widehat{\Lambda}} / I^{\prime}=\operatorname{dim}_{k} T(\Lambda)=\operatorname{dim}_{k} \Lambda+\operatorname{dim}_{k} D(\Lambda)=\operatorname{dim} 1_{\Lambda}^{m} \cdot \widehat{\Lambda},
$$

and this ends the proof of the theorem.

\section{AN APPLICATION OF A THEOREM OF S. BRENNER}

This section is devoted to a very simple interpretation of the results established in [B] in the particular case of the trivial extension $T(\Lambda)=\Lambda \ltimes D(\Lambda)$, where $\Lambda$ is an algebra such that any oriented cycle in $Q_{\Lambda}$ is zero in $\Lambda$.

We start by recalling Brenner's results.

Let $\Lambda$ be an artin algebra. An element of $\Lambda$ of the form $\alpha=f \alpha g$, where $f$ and $g$ are primitive idempotents of $\Lambda$ and $\alpha \in \mathfrak{r} \backslash \mathfrak{r}^{2}$, will be called an arrow.

Let $e$ be a primitive idempotent of $\Lambda$. A set $A$ of arrows will be called a complete set of arrows for $r e$ if

(1) it generates $\mathfrak{r} e$ (as a $\Lambda$-module).

(2) no proper subset of $A$ generates re.

A complete set of arrows for $e r$ is defined similarly.

Let $e$ be a primitive idempotent and let $\mathcal{N}$ be the set of pairs $(N, n)$ of integers such that there exist sets of arrows $A_{i}$ and $B_{i}, 0 \leq i \leq n$, of which only $A_{0}$ and $B_{0}$ can be empty, satisfying the following conditions:

(1) $i \neq j$ implies $A_{i} \cap A_{j}=\varnothing=B_{i} \cap B_{j}$.

(2) $\bigcup_{i=0}^{n} A_{i}$ is a complete set of arrows for $e r$.

(3) $\bigcup_{i=0}^{n} B_{i}$ is a complete set of arrows for $r e$.

(4) If $i \neq j$, or $i=0$, or $j=0$, then $\alpha \in A_{i}$ and $\beta \in B_{j}$ implies $\beta \alpha=0$.

(5) $\quad N=n+\operatorname{card} A_{0}$. 
Let $N_{e}=\max \{N$ : there exists $n$ with $(N, n) \in \mathcal{N}\}$ and $n_{e}=\min \left\{n:\left(N_{e}\right.\right.$, $n) \in \mathcal{N}\}$.

THEOREM 4.1 (Brenner). Let $S$ be a noninjective simple $\Lambda$-module, and let e be a primitive idempotent of $\Lambda$ such that $S \simeq \Lambda e / \mathfrak{r} e$. The middle term of the almost split sequence starting at $S$ has exactly $N_{e}$ indecomposable direct summands. Furthermore, the number of indecomposable projective direct summands is equal to $N_{e}-n_{e}$.

COROLlary 4.2 (Brenner). If $\Lambda$ is self-injective, then the number of indecomposable direct summands of $\mathfrak{r} P / \operatorname{soc} P$, where $P=\Lambda e$, is equal to $n_{e}$.

In all that follows, let $\Lambda$ be an algebra such that any oriented cycle in $Q_{\Lambda}$ is zero in $\Lambda$.

For each $h \in\left(Q_{T(\Lambda)}\right)_{0}$, let $\mathscr{C}_{h}$ be the set of oriented cycles $\mathbb{C}$ such that $\mathbb{C} \neq 0$ in $T(\Lambda)$, and $s(\mathbb{C})=e(\mathbb{C})=h$. By Corollary 3.10, we have $\mathscr{C}_{h} \neq \varnothing$.

We begin with the following definitions:

Definition 4.3. Let $\mathbb{C}, \mathbb{C}^{\prime}$ be in $\mathscr{C}_{h}$. We say that $\mathbb{C}$ and $\mathbb{C}^{\prime}$ are related, and write $\mathbb{C} \Re C^{\prime}$, if there exists an arrow $\alpha$ belonging to $\mathbb{C}$ and $\mathbb{C}^{\prime}$ with $s(\alpha)=h$ or $e(\alpha)=h$.

On the other hand, we will define a relation $\mathfrak{R}^{\prime}$ on the set

$$
A_{h}=\left\{\alpha \in\left(Q_{T(\Lambda)}\right)_{1}: e(\alpha)=h\right\} .
$$

Definition 4.4. Let $\alpha, \alpha^{\prime}$ be in $A_{h}$. We say that $\alpha$ and $\alpha^{\prime}$ are related and write $\alpha \Re^{\prime} \alpha^{\prime}$ if there exists an arrow $\beta \in\left(Q_{T(\Lambda)}\right)_{1}$ such that $\beta \alpha \neq 0$ and $\beta \alpha^{\prime} \neq 0$ in $T(\Lambda)$.

From now on, we denote by “ $\equiv$ " and “ $\approx$ " the equivalence relations generated by $\mathfrak{R}$ in $\mathscr{C}_{h}$ and by $\mathfrak{R}^{\prime}$ in $A_{h}$, respectively.

We next want to give the precise connection between these equivalence relations. For this purpose, the following results will be useful.

LEMmA 4.5. Let $\alpha_{1}, \ldots, \alpha_{m}$ be arrows in $A_{h}$, such that $\alpha_{1} \Re^{\prime} \alpha_{2} \mathfrak{R}^{\prime} \ldots \mathfrak{R}^{\prime} \alpha_{m}$. Then there exist cycles $\mathbb{C}_{1}, \ldots, \mathbb{C}_{m}$ in $\mathscr{C}_{h}$, with $\alpha_{i} \in \mathbb{C}_{i}$, for all $i=1, \ldots, m$ and $\mathbb{C}_{1} \equiv \mathbb{C}_{m}$.

Proof. We prove this by induction on $m$. Our claim clearly holds if $m=1$. Assume now that $\alpha_{1} \mathfrak{R}^{\prime} \alpha_{2} \mathfrak{R}^{\prime} \ldots \mathfrak{R}^{\prime} \alpha_{m}$, where $m \geq 2$. Then there exists $\beta \in\left(Q_{T(\Lambda)}\right)_{1}$, such that $\beta \alpha_{m-1} \neq 0$ and $\beta \alpha_{m} \neq 0$. This implies that the paths $\beta \alpha_{m-1}$ and $\beta \alpha_{m}$ belong to cycles $\mathbb{C}_{m-1}^{\prime}$ and $\mathbb{C}_{m}$, respectively. Therefore, $\mathbb{C}_{m-1}^{\prime} \mathfrak{R} \mathbb{C}_{m}$ and $\alpha_{m-1} \in \mathbb{C}_{m-1}^{\prime}, \alpha_{m} \in \mathbb{C}_{m}$.

By the induction assumption, we have cycles $\mathbb{C}_{1}, \ldots, \mathbb{C}_{m-1}$, with $\alpha_{i} \in \mathbb{C}_{i}$ for $i=1, \ldots, m-1$, and $\mathbb{C}_{1} \equiv \mathbb{C}_{m-1}$. Since $\alpha_{m-1}$ is an arrow belonging to $\mathbb{C}_{m-1}^{\prime}$ and $\mathbb{C}_{m-1}$, we have that $\mathbb{C}_{m-1} \mathfrak{R} \mathbb{C}_{m-1}^{\prime}$. Then $\mathbb{C}_{1} \equiv \mathbb{C}_{m}$, and this ends the proof of the lemma. 
Lemma 4.6. Let $\mathbb{C}_{1}, \ldots, \mathbb{C}_{m}$ in $\mathscr{C}_{h}$ such that $\mathbb{C}_{1} \mathfrak{R} \mathbb{C}_{2} \mathfrak{R} \ldots \mathfrak{R} \mathbb{C}_{m}$. Then there exist arrows $\alpha_{1}, \ldots, \alpha_{m}$ in $A_{h}$, with $\alpha_{i} \in \mathbb{C}_{i}$, for $i=1, \ldots, m$ and $\alpha_{1} \approx \alpha_{m}$.

Proof. This follows by induction on $m$, applying arguments similar to those used in the proof of Lemma 4.5.

We now give the desired connection between the equivalence relations

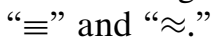

Proposition 4.7. Let $\alpha, \alpha^{\prime} \in A_{h}, \mathbb{C}, \mathbb{C}^{\prime} \in \mathscr{C}_{h}$ be such that $\alpha \in \mathbb{C}$ and $\alpha^{\prime} \in \mathbb{C}^{\prime}$. Then we have $\alpha \approx \alpha^{\prime}$ if and only if $\mathbb{C} \equiv \mathbb{C}^{\prime}$.

Proof. The result is an immediate consequence of the previous lemmas.

This readily gives the following

Corollary 4.8. $\quad \operatorname{card}\left(\mathscr{C}_{h} / \equiv\right)=\operatorname{card}\left(A_{h} / \approx\right)$.

We are now ready to describe the numbers $N_{e_{h}}$ and $n_{e_{h}}$.

Proposition 4.9. Let $h$ be a vertex in $Q_{T(\Lambda)}$, and let $e_{h}$ be the idempotent element corresponding to $h$. If $\operatorname{dim}_{k} \Lambda>1$, then $N_{e_{h}}=\operatorname{card}\left(\mathscr{C}_{h} / \equiv\right)=n_{e_{h}}$.

Proof. Consider the partition $A_{1}, \ldots, A_{t}$ induced by the relation " $\approx$ " on the set $A_{h}$. Define, for $1 \leq i \leq t, B_{i}=\left\{\beta\right.$ : there exists $\alpha \in A_{i}$ with $\beta \alpha \neq 0\}$ and $A_{0}=B_{0}=\varnothing$. Since $\operatorname{dim}_{k} \Lambda>1$, it follows that $B_{i} \neq \varnothing$, for $i=1, \ldots, t$. By construction, the pair $(t, t)$ is in $\mathcal{N}$.

We shall prove that if $(N, n) \in \mathcal{N}$, then $N=n \leq t$. In fact, let $A_{i}^{\prime}, B_{i}^{\prime}$ be sets of arrows satisfying Brenner's condition, for $0 \leq i \leq n$.

Using the above conditions and Corollary 3.10, we conclude that

(1) $B_{i}^{\prime}=\left\{\beta\right.$ : there exists $\alpha \in A_{i}^{\prime}$ with $\left.\beta \alpha \neq 0\right\}$ for $i=1, \ldots, n$.

(2) $B_{0}^{\prime}=A_{0}^{\prime}=\varnothing$.

It follows from (2) that $N=n$. On the other hand, it is easy to see that if $\alpha \approx \alpha^{\prime}$, then there exists $j$ such that $\alpha, \alpha^{\prime} \in A_{j}^{\prime}$. Then, for $1 \leq i \leq$ $t, A_{i} \subset A_{j_{i}}^{\prime}$, which implies $n \leq t$. Therefore, $N_{e_{h}}=n_{e_{h}}=\operatorname{card}\left(A_{h} / \approx\right)$, and the proof is finished by applying Corollary 4.8.

Remark 4.10. If $\operatorname{dim}_{k} \Lambda=1$, then $\operatorname{dim}_{k} T(\Lambda)=2$ and $\mathcal{N}=\{(1,0)$, $(1,1)\}$, so $N_{e_{1}}=1$ and $n_{e_{1}}=0$.

We are now in a position to restate Brenner's results in the particular case of a trivial extension $T(\Lambda)$. 
THEOREM 4.11. Let $S_{h}$ be a simple $T(\Lambda)$-module corresponding to the vertex $h$. The number of indecomposable direct summands of the middle term of the almost split sequence

$$
0 \rightarrow S_{h} \rightarrow E \rightarrow \operatorname{Tr} \mathrm{D} S_{h} \rightarrow 0
$$

is equal to the number of equivalence classes in $\mathscr{C}_{h}$. Furthermore, the number of indecomposable projective summands of $E$ is equal to zero, except if $\operatorname{dim}_{k} \Lambda=1$.

Corollary 4.12. Let $P_{h}$ be the indecomposable projective $T(\Lambda)$-module corresponding to the vertex $h$. If $\operatorname{dim}_{k} \Lambda>1$, then the number of indecomposable direct summands of $\mathfrak{r} P_{h} / \operatorname{soc} P_{h}$ is equal to the number of equivalence classes in $\mathscr{C}_{h}$.

\section{ACKNOWLEDGMENT}

The authors thank the referee for useful comments and suggestions. The authors gratefully acknowledge grant PIP 4784/96 from CONICET, Argentina.

\section{REFERENCES}

[ARS] M. Auslander, I. Reiten, and S. Smalø, "Representation Theory of Artin Algebras," Cambridge Studies in Advanced Math, Cambridge University Press, 1995.

[A] H. Asashiba, The derived equivalence classification of representation-finite selfinjective algebras, J. Algebra 214 (1999), 182-221.

[B] S. Brenner, The almost split sequence starting with a simple module, Arch. Math. 62 (1994), 203-206.

[F] E. Fernández, Extensiones Triviales y Álgebras Inclinadas Iteradas, Ph.D. thesis, Universidad Nacional del Sur, Argentina, 1999.

[FoGR] R. Fossum, P. Griffith, and I. Reiten, "Trivial Extensions of Abelian Categories," Lecture Notes in Math., Vol. 456, Springer-Verlag, Berlin-Heidelberg-New York, 1975.

[HW] D. Hughes and J. Waschbusch, Trivial extensions of tilted algebras, Proc. London Math. Soc. 46 (1983), 427-440.

[IW] Y. Iwanaga and T. Wakamatsu, "Trivial Extensions of Artin Algebras," Springer Lecture Notes, Vol. 832, 295-301, Springer, New York, 1980.

[R] M. J. Redondo, Universal Galois coverings of selfinjective algebras by repetitive algebras and Hochschild cohomology, J. Algebra 247 (2002), 332-364.

[Y] K. Yamagata, "On Algebras Whose Trivial Extensions are of Finite Representation Type,” Lecture Notes in Math., Vol. 903, 364-371, Springer-Verlag, Berlin, 1981. 